\title{
Baseline Vagal Tone Predicts BOLD Response during Elicitation of Grief
}

\footnotetext{
Mary-Frances O'Connor*,', Harald Gündel ${ }^{\mathbf{2}}$, Kateri McRae ${ }^{\mathbf{3}}$ and Richard D Lane ${ }^{\mathbf{4}}$
'UCLA Neuropsychiatric Institute, Cousins Center for Psychoneuroimmunology, LOS Angeles, CA, USA; ${ }^{2}$ Medizinische Hochschule Hannover,
Hannover, Germany; ${ }^{3}$ Department of Psychology, University of Arizona, Tucson, AZ, USA; ${ }^{4}$ Department of Psychiatry, University of Arizona,
Tucson, AZ, USA

Previous studies of the relationship between autonomic and central nervous system activity using fMRI have primarily utilized cognitive, motor or conditioning tasks. The present study investigated the association between the regional brain activity during the evocation of grief and baseline parasympathetic activity. Eight right-handed women who had experienced the death of a loved one in the past 18 months were scanned during the presentation of personalized pictures and words that evoked grief and had a measure of baseline parasympathetic activity taken. Greater posterior cingulate cortex (PCC) activity was associated with lower parasympathetic activity (eg more arousal). Connectivity has been demonstrated between the ventral PCC (vPCC) and the subgenual ACC (SACC), which then projects to the autonomic nuclei. In the present study, functional connectivity analysis revealed a positive correlation between vPCC and sACC/orbitofrontal cortical activity. Additionally, bilateral cuneus and parahippocampus were associated with higher baseline parasympathetic tone, important to visual perception in emotional processing and episodic memory respectively. Future studies should compare differences between central and peripheral arousal in complicated and non-complicated grief.

Neuropsychopharmacology (2007) 32, 2184-2 189; doi: I 0.1038/sj.npp. I 30 I342; published online I4 February 2007
}

Keywords: parasympathetic nervous system; vagus nerve; magnetic resonance imaging; emotions; bereavement; grief

\section{INTRODUCTION}

Recent neuroimaging studies have explored the relationship between peripheral autonomic activity and regional brain activity. Sympathetic activity, as measured by pupillary dilation (Critchley et al, 2005) and skin conductance responses (Nagai et al, 2004), have both been correlated with neural activation in the anterior cingulate cortex (ACC). Parasympathetic activity, as measured by the highfrequency component of heart rate variability, has also been associated with the ACC during fMRI tasks involving working memory and emotion (Gianaros et al, 2004; Lane et al, 2001; Matthews et al, 2004). The demonstrated relationship between autonomic activity indicative of arousal and ACC activity is consistent with the dense projections from the ACC to cell groups that regulate autonomic functions, such as the amygdala, hypothalamus, and brainstem nuclei (Neafsey et al, 1993). Other evidence

*Correspondence: Dr M-F O'Connor, UCLA Neuropsychiatric Institute, Cousins Center for Psychoneuroimmunology, 300 Medical Plaza, Room 3156, Los Angeles, CA 90095-7076, USA, Tel: + I 310 825 |889, Fax: + I 3107949247 ,

E-mail: mfoconnor@mednet.ucla.edu

Received 28 June 2006; revised 14 November 2006; accepted II December 2006 that arousal is associated with activity in the ACC comes from demonstrations of modulation in neural activity across stages of sleep and general anesthesia, suggesting that the activity of the ACC changes even in the absence of any overt cognitive activity (Paus, 2001).

Prior studies investigating the relationship between autonomic nervous system (ANS) activity and central nervous system (CNS) activity have been narrow in their approach. First, the tasks that have been used in demonstrating these relationships have primarily been cognitive, motor or conditioning tasks (eg Stroop, biofeedback, grip pressure, gambling, and fear conditioning). One exception is the demonstration of medial prefrontal activity associated with parasympathetic activity during emotion elicitation (Lane et al, 2001). The evocation of emotion relies on coordination of the CNS and ANS (Damasio, 1994), and very few studies have utilized emotion elicitation tasks in demonstrating the areas of brain activity that integrate these systems. Specifically, no studies examining the coordination of ANS and CNS have utilized personalized emotion stimuli. Stimuli that are personally relevant to the individual are likely to elicit strong emotion (such as pictures of family members), in part because of emotional memories. Most emotion elicited in every day life is triggered by a cue that is personally relevant (Ochsner and Gross, 2005). 
A second way in which prior studies investigating relationships between CNS and ANS activity have been narrow is using task-related designs only. Another way to investigate these ANS-CNS relationships is to consider individual differences in autonomic activity. Personality traits and genetic subtypes are associated with differential brain activity, and it may be that individual traits in basal autonomic tone are also distinctly associated with regional brain activity. Individual differences in parasympathetic activity are integral to emotion and emotion regulation (Porges, 2003). Decreased parasympathetic activity has been observed in depression (Rechlin et al, 1994), generalized anxiety disorder (Borkovec et al, 1995), and post-traumatic stress disorder (PTSD) (Sack et al, 2004). These are all conditions associated with excessively intense or prolonged emotional responses and heightened autonomic arousal.

We have previously used personalized stimuli in eliciting grief in the imaging environment (Gündel et al, 2003). To expand the understanding of the CNS-ANS relationship, the present study investigated the association between the regional brain activity during the evocation of grief in these participants and unpublished data on their baseline parasympathetic activity. We hypothesized that those individuals who show lower baseline parasympathetic activity (greater arousal) will show greater activity in the ACC.

\section{PARTICIPANTS AND METHODS}

\section{Study Participants}

Eight right-handed women who had experienced the death of a first-degree relative (eg parents and spouses) in the past year (average of 6 months) participated. They were recruited through general advertisements in hospices, hospitals, mental health clinics, and university classes. Participants were interviewed about the diagnosis, course of illness, death, and memorial of their loved one from 24 to $72 \mathrm{~h}$ before scanning. Exclusion criteria included Axis I psychiatric disorders (including current depression) evaluated by a structured, clinical interview, and medical disorders. The Human Subjects Committee at the University of Arizona in Tucson, AZ, approved the study, and participants gave written informed consent.

\section{Parasympathetic Activity Measurement}

Before the day of scanning, resting electrocardiographic (ECG) data were recorded for $5 \mathrm{~min}$. This initial recording is used in the analysis of present study; however, an additional 4 days of ECG measurement were recorded and used for reliability analysis. The ECG was recorded with a J\&J amplifier system (Poulsbo, WA), using Ag- $\mathrm{AgCl}$ electrodes, sampled at $1024 \mathrm{~Hz}$. Interbeat interval (IBI) series were derived from the raw ECG, using an R-spike detection algorithm, followed by hand screening with correction for artifacts. Heart rate variability in the high frequency band $(0.12-0.40 \mathrm{~Hz}$ ) was extracted using CMet software (Allen, 2002) as an estimate of respiratory sinus arrhythmia (RSA). RSA is a measure of parasympathetic activity, reflecting changes in beat-to-beat intervals of the heart. Variability in the $0.12-0.40 \mathrm{~Hz}$ range is under vagal control. The natural $\log$ of the variance is reported.

\section{Task Design and Stimuli}

Participants provided one photograph of their deceased loved one. The deceased was the only or central figure in the photo; any other persons were digitally removed from the photo. Photos were then matched for sex, age, and environment with one photo of a stranger. Fifteen autobiographical grief-related words were chosen from participant interviews about the death (eg collapse, funeral, and loss). These 15 words were then matched for part of speech, number of letters, and frequency of usage in the English language with 15 neutral words (eg announce, ceiling, and list).

The grief-eliciting paradigm used a $2 \times 2$ factorial design creating four conditions: (1) the deceased with grief words, (2) the stranger with grief words, (3) the deceased with neutral words, and (4) the stranger with neutral words. Each condition consisted of 15 picture-word composites or 60 composites total (Figure 1a). Comparing conditions 1 and 3 (the deceased with keywords and neutral words) with conditions 2 and 4 (the stranger with keywords and neutral words) constituted the pictorial evocation of grief. Comparing conditions 1 and 2 (the keyword with the deceased and the stranger) with conditions 3 and 4 (the neutral word with the deceased and the stranger) constituted the verbal evocation of grief.

Composites were presented on MR Vision 2000 goggles (Resonance Technology Inc.). Each picture was presented for an average of $9 \mathrm{~s}$, with a range of 7.5-10.5 s. The screen was blank during the $0.5 \mathrm{~s}$ interval between pictures. The picture-word composites were presented in a random order with regard to the four conditions. The randomization procedure was constrained so that there was an equal number of the four conditions every 20 pictures. Pictures were also counterbalanced so that half of the keywords were seen first with the deceased, and half were seen first with the stranger. The average length of presentation of each picture was also random, within the range noted above. The 60 pictures were presented continuously for a total average duration of $10.5 \mathrm{~min}$.

Immediately following scanning, each composite was rated on a scale of 1 to 10 for subjective grief. The rating was completed following scanning so that it did not contaminate emotional experience or its neural substrates. As reported in Gündel et al (2003), the four conditions differed in subjective ratings of grief and skin conductance responses $(p<0.05)$, suggesting that the different conditions reliably elicited distinct levels of grief.

\section{Data Acquisition}

Whole brain imaging data were acquired on a $1.5 \mathrm{~T}$ GE Sigma Horizon system (Milwaukee, WI). Between 19 and 22 images were collected obliquely and aligned on the AC-PC plane $(5 \mathrm{~mm}$ slice thickness, skip $=1 \mathrm{~mm}, 3.44 \times 3.44$ in plane resolution, $\mathrm{TR}=3000, \mathrm{TE}=40$, flip angle $=90$ ). The imaging data were analyzed using statistical parametric mapping (SPM2) implemented in MATLAB (Mathworks Inc., Sherborn, MA, USA). Automated algorithms were used 

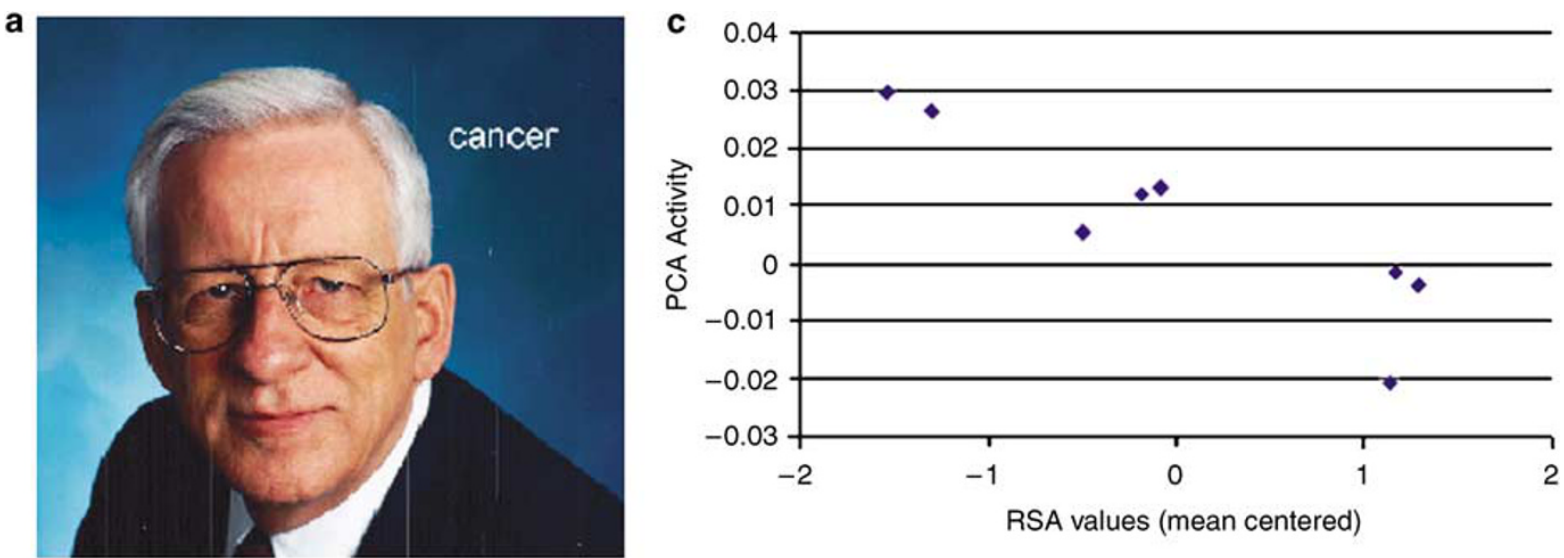

b

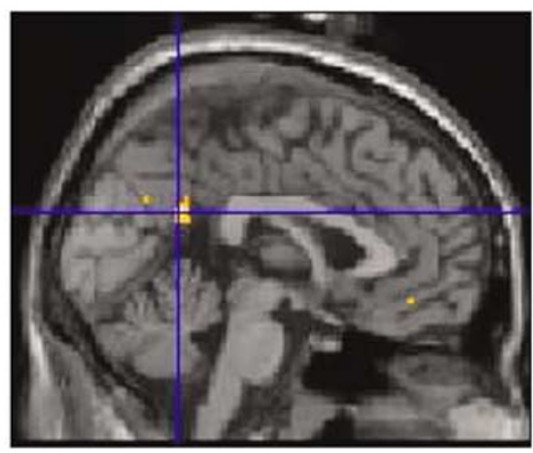

d

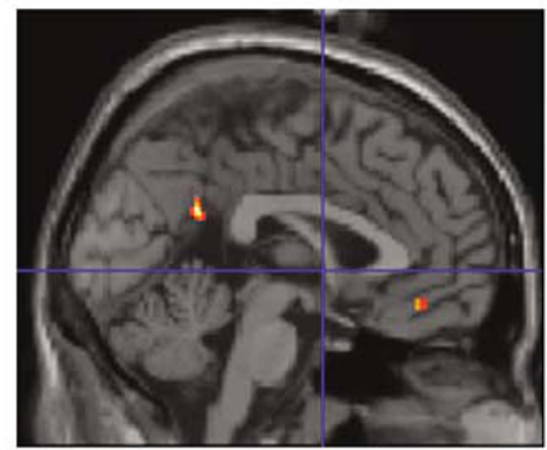

Figure I (a) Photo of the deceased with grief-related word. (b) Saggital view of the vPCC (Tailarach and Tournoux coordinates: 0, -54, 24). (c) Scatterplot of the negative relationship between VPCC activity and RSA (increased VPCC, decreased RSA) in the Deceased $>$ Stranger condition. VPCC, ventral posterior cingulate cortex; RSA, respiratory sinus arrhythmia. (d) Brain regions resulting from functional connectivity analysis revealing positively correlated activity between the sACC/orbitofrontal cortex and posterior cingulate activity.

to align each subject's sequential MRI images, spatially normalized into the stereotactic space of Tailarach and Tournoux (1988) using the 305 MNI (Montreal Neurological Institute) standard brain and smooth (8 $\mathrm{mm}$ FWHM) images.

Analysis was carried out using the general linear model in an event-related analysis. Subject-specific low-frequency drift was removed by a high-pass filter and global signal changes were removed by including a global covariate. Effects at each voxel were estimated and regionally specific effects were compared using linear contrasts. We investigated regional BOLD changes independent of variations in whole brain measurements, and generated separate normalized $t$-score (ie $Z$ score) maps of BOLD increases during each of the four conditions. These $t$-statistics constituted a statistical parametric map (SPM $\{Z\})$. Statistical inferences were based on a theory of random Gaussian fields. Contrasts for individual subjects were aggregated for the group in a random effects analysis. BOLD changes were considered statistically significant if they equaled or exceeded $p<0.001$, uncorrected.

\section{Data Analysis}

The imaging data were first analyzed for main effects and are reported in Gündel et al (2003). For each participant, images taken during the Deceased and Stranger photos and images taken during the Neutral and Grief-related words were modeled as events. After the task was modeled for each participant, planned comparisons were computed as linear contrasts to investigate Deceased $>$ Stranger activity, and Grief Word $>$ Neutral Word activity. To assess correlations between individual differences in RSA and neural activity, the measure of (mean-corrected) RSA was entered as a regressor into each random effects group analysis (Deceased $>$ Stranger and Grief Word $>$ Neutral Word). BOLD changes were considered statistically significant if they equaled or exceeded $p<0.001$, corrected for multiple comparisons at the cluster level $(p<0.05)$.

Follow-up analyses examined functional connectivity with the posterior cingulate activation for the Deceased $>$ Stranger contrast. Average values were extracted from each individual's Deceased $>$ Stranger contrast image using a region of interest defined by the posterior cingulate cluster identified in the whole-brain contrast. These values were entered as a regressor into a random-effects group analysis with the same contrast to identify regions that covary with posterior cingulate activity across individuals.

\section{RESULTS}

\section{Behavior and Physiology}

The RSA values used reliably correlated with 4 days of baseline RSA recording subsequent to the imaging day $(p<0.01)$, indicating that these values represent a tonic level of parasympathetic activity. Those participants with higher tonic RSA had lower subjective reports of grief during 
scanning (in a comparison of the grief and neutral conditions) (RSA $(6.16 \pm 1.11)$, subjective rating of grief $(5.9 \pm 2.06) ; r=-0.74, p<0.05)$.

\section{Brain Imaging}

As previously published, the main contrasts showed three brain regions independently activated by the Deceased $>$ Stranger and Grief Word $>$ Neutral Word contrasts: PCC, medial/superior frontal gyrus, and cerebellum. Distinct regions were also activated by the two contrasts: Deceased $>$ Stranger included the cuneus, superior lingual gyrus, insula, dorsal ACC, inferior temporal gyrus, and fusiform gyrus; Grief Word $>$ Neutral Word included the precuneus, precentral gyrus, midbrain, and vermis.

In the regression analyses of the present study, using the RSA covariate with the activation of Deceased $>$ Stranger, a positive relationship was found with the right cuneus, and a negative relationship was found with the right PCC (Table 1 and Figure $1 \mathrm{~b}$ ). Using the RSA covariate with the activation of Grief Word $>$ Neutral Word, a positive relationship was found with the left cuneus and PHG. A scatterplot demonstrates that this effect was not due to outliers (Figure 1c).

To test the association between PCC and subgenual ACC (sACC) in the present study, a functional connectivity analysis that used the PCC activation $(x=0, y=-54$, $z=24$ ) as a seed region was conducted. This analysis revealed activity in the sACC/orbitofrontal cortex $(x-2$, $y=40, z=-14, z=4.01$ ) that positively correlated with posterior cingulate activity in the Deceased $>$ Stranger contrast, supporting our conclusion (Figure 1d).

\section{DISCUSSION}

The present study investigated the overlap between those individuals with higher baseline parasympathetic activity and brain activation during a personalized emotioneliciting task. The results did not support our hypothesis that ACC would be negatively associated with individuals

Table I Brain Region Activity Associated with Respiratory Sinus Arrhythmia (RSA) during Grief Elicitation $(N=8)$

\begin{tabular}{lccc}
\hline Region & $\begin{array}{c}\text { Coordinates } \\
(\mathbf{x}, \mathbf{y}, \mathbf{z})\end{array}$ & $\begin{array}{c}\text { Cluster } \\
\text { size }(\boldsymbol{p})\end{array}$ & Z-score \\
\hline $\begin{array}{l}\text { (A) Areas of negative correlation between } \\
\text { regional activity, decreased RSA) } \\
\text { Ventral posterior cingulate cortex }>\text { Stranger and RSA (increased }\end{array}$ & $0,-54,24$ & $42(0.045)$ & 3.81
\end{tabular}

(B) Areas of positive correlation between Deceased > Stranger and RSA (increased regional activity, increased $R S A$ )

$$
\begin{array}{llll}
\text { Cuneus } & 20,-72,8 & 57(0.009) & 5.04
\end{array}
$$

(C) Areas of positive correlation between Grief Word > Neutral Word and RSA (increased regional activity, increased RSA)

$$
\begin{array}{llll}
\text { Cuneus } & -16,-84,24 & 102(0.001) & 4.51 \\
\text { Parahippocampal gyrus } & -28,-4,-18 & 36(0.043) & 4.08
\end{array}
$$

with lower parasympathetic activity (greater baseline arousal). Instead, PCC activity was negatively associated with individual differences in parasympathetic activity in the context of grief elicitation. Although unexpected, this is an exciting new result, as recent projection studies have demonstrated specific connectivity between the PCC and the sACC (Vogt et al, 2006), which projects to the autonomic nuclei in the brainstem (Neafsey et al, 1993).

To the extent that arousal is associated with ACC, the task conditions that activate the ACC in prior studies are relevant. The stimulus conditions in the present experiment strongly pull for memories - the grief-related words are targeted for specific experiences in the past. This is in contrast to other studies that involve emotion $\times$ attention interactions (eg focusing attention on feeling, or feelings capturing attention) or regulation of emotion (Ochsner et al, 2002) that involve ACC. Our original hypothesis was that we would observe ACC activity associated with tonic vagal tone based on prior studies; however, regional brain activity is always a product of the nature of the experimental conditions, and the experimental conditions in the present study differed importantly by eliciting personal emotional memories.

PCC activity is elicited by an interaction between emotion and memory (Maddock et al, 2003), specifically by the arousal dimension of emotional stimuli, and is important in monitoring the environment for emotionally salient events (Vogt et al, 1992). Comparison of these environmental events to memories utilizes the PCC (as suggested by its dense interconnections with the hippocampus and its role in the acquisition of familiarity; Kosaka et al, 2003).

Increased PCC activity has been demonstrated in participants who had experienced a bank robbery when the robbery video was contrasted with a control video (Fischer et al, 1996). PCC activity has also been shown in studies of participants with PTSD during an autobiographical trauma script, as compared to those without PTSD (Lanius et al, 2004). In the present study, the environmental cue (eg photo of loved one or autobiographical grief-related word) triggers retrieval of an emotional memory (eg the death event) in those with lower parasympathetic activity (eg more arousal).

The coordinates of the PCC in the present study clearly place it in the vPCC. Recent projection maps have shown that there are functional differences between the dorsal PCC (dPCC) and the ventral PCC (vPCC; Vogt et al, 2006). Vogt and colleagues propose a division between dPCC, which orients the body in space via the cingulate motor areas, and the vPCC, which interacts with sACC to process self-relevant emotional and nonemotional information and objects. The reciprocal connection between vPCC and ACC may explain the indirect association between the vPCC and lower parasympathetic autonomic activity in the present study. This is supported by the data from our functional connectivity analysis, demonstrating that activity in the sACC/orbitofrontal region is correlated with activity in the vPCC.

Those individuals with higher baseline RSA showed more cuneus activation in a covariate analysis with both the Deceased $>$ Stranger contrast, and Grief Word $>$ Neutral Word contrast. The cuneus is a region activated during tasks requiring emotional processing and visual perception, 
such as emotion induction elicited with visual stimuli (Ganis et al, 2004). We observed a correlation with right cuneus in the context of the Deceased $>$ Stranger contrast and a correlation with left cuneus in association with the Grief Word $>$ Neutral Word contrast. Given the hemispheric specialization that distinguishes visual and verbal tasks, the visual association areas activated may have been lateralized based on the nature of the exteroceptive input.

Those with higher baseline RSA showed more parahippocampal (PHG) activity with the Grief Word $>$ Neutral Word contrast. The PHG is part of the hippocampal complex that underlies episodic memory (Squire et al, 2004). An earlier finding of a negative correlation between blood pressure and PHG activity has been interpreted to mean that the PHG and other medial lobe structures 'preferentially represent states of low sympathetic arousal and high parasympathetic tone (McEwen and Sapolsky, 1995, p 268)'. Our finding of PHG activation during high parasympathetic tone dove tails nicely with data.

\section{CONCLUSION}

The present study demonstrates greater activity in the vPCC in response to grief stimuli in individuals with lower baseline parasympathetic activity. This result lends support to the hypothesis that the vPCC processes self-relevant emotional information. The connection to sACC, and its role in autonomic arousal, may explain the association between the vPCC activity and lower baseline parasympathetic tone. Additionally, activity in the right and left cuneus and PHG were associated with higher baseline parasympathetic tone.

One limitation of the present study may be that the vagal tone was not measured during the grief-eliciting task in the scanner; however, we attempted to overcome this limitation by measuring the participants' RSA on multiple days to increase reliability of the vagal tone measure and therefore the likelihood that it would be very similar during scanning. Additional information could have been added by including a measure of sympathetic activity. Although other investigators have used the low-frequency component of heart rate variability as a proxy for sympathetic activity, there is controversy surrounding this usage (Malik et al, 1996). Consequently, we elected not to use it in the current study. Another limitation is the small number of participants; however, we used a conservative significance level $(p<0.001)$ and corrected for multiple comparisons to reduce the possibility that these observations were due to chance. In addition, the brain regions elicited were specific and distinct, rather than widespread activation. As this is the first study to examine at the peripheral and central arousal during grief elicitation it cannot be considered definitive, but rather informs future research through the development of testable hypotheses.

Future clinical research in this area should consider this individual physiological difference in comparing those with complicated and non-complicated grief (Prigerson et al, 1995). Peripheral parasympathetic arousal is associated with individual differences in grief response patterns (O'Connor et al, 2005), such that higher tonic vagal activity is associated with adaptation to bereavement. Comparing the associations between central and peripheral arousal in complicated and non-complicated grief may elucidate the neural correlates of those individual differences.

\section{ACKNOWLEDGEMENTS}

We thank John JB Allen for RSA analysis assistance and Carolyn L Fort for MRI analysis assistance. Mary-Frances O'Connor is a Friends of the Semel Institute Research Fellow.

\section{REFERENCES}

Allen JJB (2002). Calculating metrics of cardiac chronotropy: a pragmatic overview. Psychophysiology 39: S18.

Borkovec TD, Roemer L, Kinyon J (1995). Disclosure and worry: opposite sides of the emotional processing coin. In: Pennebaker JW (ed). Emotion, Disclosure and Health. American Psychological Association: Washington, DC. pp 47-70.

Critchley HD, Tang J, Glaser D, Butterworth B, Dolan RJ (2005). Anterior cingulate activity during error and autonomic response. NeuroImage 27: 885.

Damasio AR (1994). Descartes' Error: Emotion, Reason, and the Human Brain. Putnam Publishing Group: New York.

Fischer H, Wik G, Fredrikson M (1996). Functional neuroanatomy of robbery re-experience: affective memories studied with PET. Neuroreport 7: 2081-2086.

Ganis G, Thompson WL, Kosslyn SM (2004). Brain areas underlying visual mental imagery and visual perception: an fMRI study. Cogn Brain Res 20: 226-241.

Gianaros PJ, Van der Veen FM, Jennings JR (2004). Regional cerebral blood flow correlates with heart period and highfrequency heart period variability during working-memory tasks: implications for the cortical and subcortical regulation of cardiac autonomic activity. Psychophysiology 41: 521-530.

Gündel H, O'Connor M-F, Littrell L, Fort C, Lane R (2003). Functional neuroanatomy of grief: an fMRI study. $A m J$ Psychiatry 160: 1946-1953.

Kosaka H, Omori M, Iidaka T, Murata T, Shimoyama T, Okada T et al (2003). Neural substrates participating in acquisition of facial familiarity: an fMRI study. Neuroimage 20: 1734-1742.

Lane R, Reiman E, Ahern GT, Thayer J (2001). Activity in medial prefrontal cortex correlates with vagal component of heart rate variability during emotion. Brain Cogn 47: 97-100.

Lanius RA, Williamson PC, Densmore M, Boksman K, Neufeld RW, Gati JS et al (2004). The nature of traumatic memories: a 4-T fMRI functional connectivity analysis. Am J Psychiatry 161: $36-44$.

Maddock RJ, Garrett AS, Buonocore MH (2003). Posterior cingulate cortex activation by emotional words: fMRI evidence from a valence decision task. Hum Brain Mapp 18: 30-41.

Malik M, Bigger JT, Camm AJ, Kleiger RE, Malliani A, Moss AJ et al (1996). Heart rate variability: standards of measurement, physiological interpretation, and clinical use. Eur Heart J 17: 354-381.

Matthews SC, Paulus MP, Simmons AN, Nelesen RA, Dimsdale JE (2004). Functional subdivisions within anterior cingulate cortex and their relationship to autonomic nervous system function. NeuroImage 22: 1151.

McEwen BS, Sapolsky RM (1995). Stress and cognitive function. Curr Opin Neurobiol 5: 205-216.

Nagai Y, Critchley HD, Featherstone E, Trimble MR, Dolan RJ (2004). Activity in ventromedial prefrontal cortex covaries with sympathetic skin conductance level: a physiological account of a 'default mode' of brain function. NeuroImage 22: 243. 
Neafsey E, Terreberry R, Hurley K, Ruit K, Frysztak R (1993). Anterior cingulate cortex in rodents: connections, visceral control functions, and implications for emotion. In: Vogt BA, Gabriel M (eds). Neurobiology of Cingulate Cortex and Limbic Thalamus: A Comprehensive Handbook. Birkhauser: Boston.

O'Connor M-F, Allen JJB, Kaszniak AW (2005). Emotional disclosure for whom? a study of vagal tone in bereavement. Biol Psychol 68: 135-146.

Ochsner KN, Bunge SA, Gross JJ, Gabrieli JDE (2002). Rethinking feelings: an fMRI Study of the Cognitive Regulation of Emotion. J Cogn Neurosci 14: 1215-1229.

Ochsner KN, Gross JJ (2005). The cognitive control of emotion. Trends Cogn Sci 9: 242.

Paus T (2001). Primate anterior cingulate cortex: where motor control, drive and cognition interface. Nat Rev Neurosci 2: SW (2003). The Polyvagal Theory: phylogenetic contributions to social behavior. Physiol Behav 79: 503.

Prigerson HG, Frank E, Kasl SV, Reynolds III CF, Anderson B, Zubenko GS et al (1995). Complicated grief and bereavement- related depression as distinct disorders: preliminary empirical validation in elderly bereaved spouses. Am J Psychiatry 152: 22-30.

Rechlin T, Weis M, Spitzer A, Kaschka WP (1994). Are affective disorders associated with alterations of heart rate variability? $J$ Affect Disord 32: 271-275.

Sack M, Hopper JW, Lamprecht F (2004). Low respiratory sinus arrhythmia and prolonged psychophysiological arousal in posttraumatic stress disorder: heart rate dynamics and individual differences in arousal regulation. Biol Psychiatry 55: 284.

Squire LR, Stark CE, Clark RE (2004). The medial temporal lobe. Annu Rev Neurosci 27: 279-306.

Tailarach J, Tournoux P (1988). Coplanar Stereotactic Atlas of the Human Brain. George Thieme Verlag: Stuttgart.

Vogt BA, Finch DM, Olson CR (1992). Functional heterogeneity in cingulate cortex: the anterior executive and posterior evaluative regions. Cereb Cortex 2: 435-443.

Vogt BA, Vogt L, Laureys S (2006). Cytology and functionally correlated circuits of human posterior cingulate areas. Neuroimage 29: 452. 